\section{A program to assess baroreceptor cardiac reflex function}

\author{
GUSTAVO A. REYES DEL PASO \\ University of Jaén, Jaén, Spain
}

This paper describes a computer program for the noninvasive evaluation in the time domain of the part of the baroreceptor reflex that relates blood pressure to interbeat interval from spontaneous hemodynamic patterns in which the reflex is operating. The program obtains the sensitivity of the baroreceptor reflex (slope of the regression line between the interbeat interval and systolic blood pressure in $\mathrm{msec} / \mathrm{mmHg}$ ) and an index of the baroreceptor power to regulate cardiac activity. These statistics are obtained differentially both when the systolic blood pressure is increasing and when it is decreasing. The results obtained by using this software during a mental load task were compared with those obtained through spectral analysis.

The baroreceptor reflex that relates blood pressure to interbeat interval and other hemodynamic parameters is a basic mechanism for the regulation of blood pressure variability and a powerful source of vagal afferent input to the central nervous system (Guyton, 1984). There is evidence linking it to the loss of parasympathetic cardiac control in subjects with cardiovascular disorders (Eckberg, 1980b). Some data also suggest that this mechanism could play some role in human essential hypertension (McCubbin, Green, \& Page, 1956; Steptoe \& Sawada, 1989), since the reflex is supressed in hypertensive subjects (Bertinieri et al., 1987; Gribbin, Pickering, Sleight, \& Peto, 1971). It is known that the baroreceptor cardiac reflex function is sensitive to psychological manipulations, reducing its sensitivity (expressed as the change in interbeat interval per unit change in systolic blood pressure) during mental stress (Robbe et al., 1987; Steptoe \& Sawada, 1989).

Until recently the sensitivity of the baroreceptor reflex was evaluated by means of two methods that produced experimental alterations of the cardiovascular function. The oldest method is a pharmacological technique in which interbeat interval and systolic blood pressure responses caused by intravenous injection of a pressor agent, normally phenylephrine, are measured (Smyth, Sleight, \& Pickering, 1969). The other is the neck chamber technique, in which the baroreceptors are mechanically stimulated by suction (Eckberg, Cavanaugh, Mark, \& Abboud, 1975). Both methods are obtrusive, changing the system being assessed and, therefore, limiting their ability to de-

This research was supported by a Grant of the Commission of the European Communities Medical and Health Research Program for the concerted action "Quantification of Parameters for the Study of Breakdown in Human Adaptation." I would also like to thank L. J. M. Mulder and his colleagues. Correspondence should be addressed to G. A. Reyes del Paso, Departamento de Personalidad, Evaluación y Tratamiento Psicológico, Facultad de Humanidades, Universidad de Jaén, 23071 Jaén, Spain. tect natural variations in function. With the availability of equipment that allows the continuous and noninvasive measurement of blood pressure, following the principle of Peñáz (Reyes, 1991; Settels \& Wesseling, 1985), two new methods have been developed to evaluate the sensitivity of the baroreceptor reflex in a noninvasive way from the spontaneous covariation of systolic blood pressure and interbeat interval: one in the frequency domain and the other in the time domain. The first is based on spectral analysis, in which the modulus or gain function between the variations in systolic blood pressure and interbeat interval generates an estimate of the baroreceptor sensitivity that correlates with the pharmacological method (Robbe et al., 1987). The second method, which was developed by Bertinieri et al. (1985), still uses invasive measures of blood pressure and consists of the identification of cardiac sequences in which the baroreflex is operating. To do this, sequences of consecutive cardiac cycles are sought in which systolic blood pressure increase is accompanied by an increase in interbeat interval or in which a decrease in systolic blood pressure is accompanied by a decrease in interbeat interval. The regression line between the systolic blood pressure values and the interbeat interval values produces an estimate of baroreceptor cardiac reflex sensitivity in $\mathrm{msec} / \mathrm{mmHg}$. At the same time, the number of cycles contributing to sequences in a measurement period reflects the relative power of the baroreflex to regulate cardiac activity. This method also allows us to differentiate the sensitivity and the power of the baroreceptor reflex in terms of increases or decreases in the systolic blood pressure. This might reflect differences in the physiological control mechanisms. Concretely, there have been reports indicating that the sympathetic and the vagal determinants of heart rate regulation are different when blood pressure rises as opposed to when it falls (Eckberg, 1980a; Miyajima, Matsukawa, Yamada, Tochikubo, \& Kanedo, 1986; Pickering, Gribbin, \& Sleight, 1972; Scher \& Young, 1970; Thames \& Kontos, 1970). Following these observations, unpublished data from our laboratory using invasive vagal measures shows that the "up" sequences are better predictors of vagal cardiac activity than are the "down" sequences. This method appears promising and well-suited for psychophysiology investigations, because measures can be obtained unobtrusively. In this paper, a computer program for the analysis of baroreceptor cardiac reflex function, following the time domain method developed by Bertinieri et al. (1985), is described.

\section{Description}

The program developed is written in BASIC and compiled to run under MS-DOS. The input file format consists of a short heading, with information regarding experimental ID, duration of the trial, and number of cardiac cycles included in the file and followed by the beat-by-beat values of interbeat interval and systolic blood pressure. These 
beat-by-beat values have to be corrected for artifacts by linear interpolation or deletion before running the program. On starting, the program asks for the subject's ID, followed by the number of the trial to be analyzed (datafile names consist of subject ID and trial number). The program searches the file for sequences of three to six consecutive cardiac cycles in which the systolic blood pressure increases (by at least $1 \mathrm{mmHg}$ in each beat) in combination with an increase in the interbeat interval (of at least $2 \mathrm{msec}$ in each beat), or sequences in which the decrease of the systolic blood pressure is accompanied by a decrease in the interbeat interval (following the same criteria of minimum change). When one of these sequences is detected, the correlation between the variables is computed along with the corresponding regression line. The sensitivity of the reflex is expressed as a change in the interbeat interval (in milliseconds) for each $\mathrm{mmHg}$ change in systolic blood pressure and is measured by the slope of the regression line. These statistics are calculated for all sequences in the trial. The program graphically displays the record of interbeat interval and systolic blood pressure, marking the initial and final points of each sequence detected. For each file, the program obtains, displays on screen, and stores to disk the sensitivity of the baroreflex, the intersection with the coordinated axis values, the correlation between the systolic blood pressure and interbeat interval, the proportion (in percent) of cardiac cycles that form part of the sequences for the acquisition of the previous parameters with respect to the total number of cycles during the analysis period (i.e., baroreceptor power), and the number of sequences of six, five, four, and three cardiac cycles detected. All of these parameters are obtained both when the systolic blood pressure is increasing and when it is decreasing.

\section{Testing the Program}

The time domain analysis technique, unlike spectral analysis, could be applied to experimental tasks with both phasic and tonic response paradigms and with both short and long time periods. The statistical requirement of stationarity-implicit in the spectral analysis techniquewhich demands a tonic response paradigm with a long measurement period (about several minutes of beat-bybeat values of interbeat interval and blood pressure), is not a requirement for the time domain technique. A minimum of a few seconds could theoretically be sufficient to obtain an estimate of baroreceptor reflex sensitivity. However, the validity of such an estimate would need to be tested against the traditional pharmacological or spectral analysis methods. Our program was compared with the spectral analysis method applied to a typical mental load task with a tonic response paradigm and a long measurement period. This type of task is frequently used in psychophysiological research to study cardiovascular reactivity to cognitive stressors. The validity of the time domain method in tasks in which spectral analysis is not applicable will probably require specific comparisons with the traditional pharmacological method.
The program and method were tested in a study in which 15 subjects performed a mental load task of 5-min duration after a 10-min rest period. A memory search and counting task were used for the mental load condition. The subjects had to memorize four letters presented on a computer screen (memory set). Then, single letters appeared on the screen at 3-sec intervals, with the subject required to decide whether or not the letter belonged to the memory set. If the decision was affirmative, the subject had to press a button with the right hand. At the same time, the subject had to count mentally the number of times each letter of the memory set appeared on the screen. Blood pressure was recorded continuously with the noninvasive FIN.A.PRES device (Settels \& Wesseling, 1985) from the third fingertip of the left hand. The hand was positioned at the level of the apex cordis. Interbeat interval was recorded from Lead II of the electrocardiograph. The results obtained were then compared with those found through spectral analysis using the commercially available program CARSPAN (Mulder, van Dellen, van der Meulen, \& Opheikens, 1988), which had been validated in comparison with the pharmacological method (Robbe et al., 1987). Because both programs use the same input data files, the time domain program was adapted to work with the CARSPAN input format (Mulder et al., 1988).

The results are shown in Table 1. As can be seen, both baroreceptor reflex sensitivity and power are reduced during the mental load task compared with the baseline period, all of these differences being significant at the .01 level. The decrease in the baroreceptor reflex sensitivity during the mental load task is supported by the findings of Steptoe and Sawada (1989), who used a mental arithmetic task. In addition to that study, in the present experiment, greater baroreceptor sensitivity was obtained in the "up" sequences than in the "down" sequences (these differences being significant in the baseline period), confirming research in cats by Bertinieri et al. (1985) and findings by other authors (Mancia et al., 1983). Enlarging on the study of Steptoe and Sawada, the power of the baroreceptor reflex to regulate cardiovascular activity was analyzed in the present experiment. In a way similar to that of reflex sensitivity, the power also decreases during the mental load task.

Table 2 shows the correlations between the baroreceptor reflex sensitivity obtained in the time domain and the modulus function of the spectral analysis in the middlefrequency $(.07-.14 \mathrm{~Hz})$ and high-frequency $(.14-.40 \mathrm{~Hz})$ bands. As can be seen, the correlations are high, being

Table 1

Baroreceptor Reflex Sensitivity (BRS) and Power (BRP) Mean Values During Baseline and Mental Load Task

\begin{tabular}{lcl}
\hline & Baseline & Task \\
\hline BRS (Up) & 23 & 16 \\
BRS (Down) & 18.5 & 15 \\
BRP (Up) & 18.2 & 10.6 \\
BRP (Down) & 21.7 & 10.8 \\
\hline
\end{tabular}


Table 2

Correlations Between Baroreceptor Reflex Sensitivity

Obtained in the Time Domain and the Modulus Function of the Spectral Analysis in the Middle (.07-.14 Hz) and High (.14-.40 Hz) Frequency Bands

\begin{tabular}{|c|c|c|c|c|}
\hline \multirow[b]{2}{*}{ Frequency } & \multicolumn{2}{|c|}{ Baseline } & \multicolumn{2}{|c|}{ Task } \\
\hline & Up & Down & Up & Down \\
\hline Middle & .86 & .80 & .72 & .40 \\
\hline High & .95 & .94 & .80 & .72 \\
\hline
\end{tabular}

a little greater for the high-frequency band than for the middle-frequency band. It is interesting that the correlations are reduced during the mental-load-task period, when the signal stationarity assumption of the spectral analysis could be violated. To calculate reliable spectral indexes, there should be no slow nonrhythmic changes in the data nor variability fluctuations (the mean and the variance of the time series to be analyzed should remain constant from one segment to another). However, the phasic nature of reaction patterns during laboratory stressors may violate this assumption. Figure 1 shows the changes in interbeat interval and systolic blood pressure during the mental load task with respect to baseline. The response patterns clearly show a nonstationarity trend in the data that could produce errors in the spectral index. Also related to the signal-stationarity assumption of the spectral analysis is the need to use relatively long sampling periods (more than $4 \mathrm{~min}$ ) in order to obtain reliable results. Free of the statistical requirements of spectral methods, the time domain technique has the potential advantage of permitting baroreflex analysis over phasic tasks and short time periods (Steptoe \& Sawada, 1989).

\section{Program Availability}

This software can be obtained by electronic mail (castellar@ugr.es) or by writing to Gustavo A. Reyes del Paso, Departamento de Personalidad, Evaluación y Tratamiento Psicológico, Facultad de Humanidades, Universidad de Jaén, 23071 Jaén, Spain.

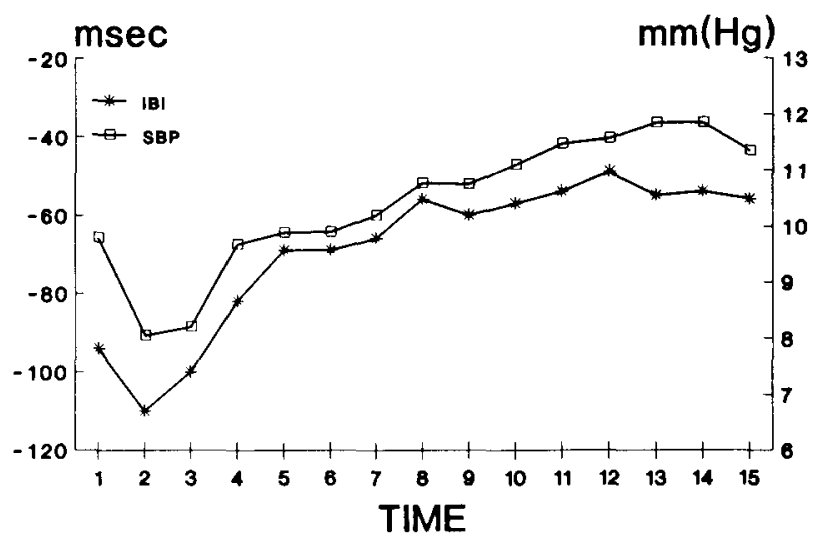

Figure 1. Interbeat interval (IBI) and systolic blood pressure (SBP) during the mental load task. The data are plotted as 13.3-sec means for the first $200 \mathrm{sec}$ of the task in differential scores with respect to the average baseline.

\section{REFERENCES}

Bertinieri, G., di Rienzo, M., Cavallazzi, A., Ferrari, A. U., Pedotti, A., \& Mancia, G. (1985). A new approach to analysis of the arterial baroreflex. Joumal of Hypertension, 3(Suppl. 3), S79-S81.

Bertinieri, G., di Rienzo, M., Parati, B., Pomidossi, G., PeDOTTI, A., ZANCHETTI, A., \& MANCIA, G. (1987). Baroreceptor-heart rate reflex studied in normotensive and essential hypertensives by beatto-beat analysis of 24-hour blood pressure and heart rate. Journal of Hypertension, 5(Suppl. 5), 5333-5335.

ECKBERG, D.L. (1980a). Nonlinearities of the human carotid baroreceptor-cardiac reflex. Circulation Research, 47, 208-216.

ECKBeRG, D. L. (1980b). Parasympathetic cardiac control in human disease: A critical review of methods and results. American Journal of Physiology, 239, H581-H593.

Eckberg, D. L., Cavanaugh, M. S., Mark, A. L., \& Abboud, F. M. (1975). A simplified neck suction device for activation of carotid baroreceptors. Journal of Laboratory \& Clinical Medicine, 85, 167-173.

Gribiin, B., Pickering, T. G., Sleight, P., \& Peto, R. (1971). Effect of age and high blood pressure on baroreflex sensitivity in man. Circulation Research, 29, 424-431.

Guyton, A. C. (1984). Tratado de Fisiología Médica. Mexico City: Interamericana.

Mancia, G., Ferrari, A., Gregorini, L., Parati, G., Pomidossi, G., Bertinieri, G., Grassi, G., di Rienzo, M., Pedotti, A., \& ZanCHETTI, A. (1983). Blood pressure and heart rate variabilities in normotensive and hypertensive human beings. Circulation Research, 53, 96-104.

McCubbin, J. W., Green, J. H., \& PAge, I. H. (1956). Baroreceptor function in chronic renal hypertension. Circulation Research, 4, 205-210.

Miyajima, E., Matsukawa, T., Yamada, Y., Tochikubo, O., \& KANEDO, Y. (1986). Differential baroreflex changes in sympathetic and parasympathetic nerve activities in patients with essential hypertension. Journal of Hypertension, 4, S219-S221.

Mulder, L. J. M., van Dellen, H. J., van der Meulen, P., \& OPHEIKENS, B. (1988). CARSPAN: A spectral analysis program for cardiovascular time series. In F. J. Maarse, L. J. M. Mulder, W. Sjouw, \& A. Akkerman (Eds.), Computers in psychology: Methods, instruments \& psychodiagnostics (pp. 30-38). Lisse, The Netherlands: Swets \& Zeitlinger.

Pickering, T.G., Gribbin, B., \& Sleight, P. (1972). Comparison of the reflex heart rate response to rising and falling arterial pressure in man. Cardiovascular Research, 6, 277-283.

REYEs, G. A. (1991). The continous and non-invasive recording of blood pressure following the Peñáz method: A computer programme. Evaluación Psicologica/Psychological Assessment, 7, 369-375.

Robbe, H. W. J., Mulder, L. J. M., Rüddel, H., Veldman, J. B. P., LANGEWITZ, W. A., \& MuLDER, G. (1987). Assessment of baroreflex sensitivity by means of spectral analysis. Hypertension, 10, 538-543.

Scher, A. M., \& Young, A. C. (1970). Reflex control of heart rate in the unanesthetized dog. American Journal of Physiology, 218, 780-789.

Settels, J. J., \& Wesseling, K. H. (1985). FIN.A.PRES.: Noninvasive finger arterial pressure waveform registration. In J. Orlebeke, G. Mulder, \& L. Doornen (Eds.), Psychophysiology of cardiovascular control (pp. 267-283). New York: Plenum Press.

Smyth, H. S., Sleight, P., \& Pickering, G. W. (1969). Reflex regulation of arterial pressure during sleep in man: A quantitative method of assessing baroreflex sensitivity. Circulation Research, 24, 109-121.

STEPTOE, A., \& SaWAda, Y. (1989). Assessment of baroreceptor reflex function during mental stress and relaxation. Psychophysiology, 26, 140-147.

Thames, M. D., \& Kontos, H. A. (1970). Mechanisms of baroreceptorinduced changes in heart rate. American Journal of Physiology, 218, 251-256.

(Manuscript received December 16, 1991; revision accepted for publication May 28, 1993.) 